Service social

\title{
L'univers des personnes âgées et les technologies nouvelles : Recension des écrits
}

\section{Étienne LaBillois et Nicole Boucher}

Volume 41, numéro 1, 1992

L'avenir des services ou services d'avenir

URI : https://id.erudit.org/iderudit/706559ar

DOI : https://doi.org/10.7202/706559ar

Aller au sommaire du numéro

\section{Éditeur(s)}

École de service social de l'Université Laval

ISSN

1708-1734 (numérique)

Découvrir la revue

Citer cet article

LaBillois, É. \& Boucher, N. (1992). L’univers des personnes âgées et les technologies nouvelles : Recension des écrits. Service social, 41(1), 84-103. https://doi.org/10.7202/706559ar

\section{Résumé de l'article}

Le phénomène du vieillissement démographique s'accompagne au Québec comme ailleurs dans nos sociétés occidentales, d'une révolution technologique. Or, faute de recherche en ce domaine, on connaît mal comment les personnes âgées sont touchées par ces technologies nouvelles. Se fondant sur une littérature essentiellement américaine ou européenne, les auteurs ont relevé trois secteurs où l'impact des nouvelles technologies sur la population âgée est majeur. Il s'agit du travail, de la santé et des loisirs.

Sur le plan du travail, l'introduction de la technologie informatique semble provoquer l'accélération du vieillissement professionnel et la mise au « rencart » des anciens. Ainsi, à 45 ans, on est maintenant classé comme travailleur " âgé ", éventuellement " recyclable », sinon remplacé par des jeunes disposant d'une formation supérieure. La déqualification de la main-d'oeuvre âgée par suite de la modernisation des entreprises semble donc un enjeu majeur.

Par contre, sur le plan de la santé et des loisirs, les personnes âgées bénéficient maintenant de technologies" adaptées " permettant de compenser leur handicap ou de s'amuser de façon stimulante et originale. La consommation de ces nouveaux produits par les personnes âgées est fortement encouragée et apparemment souvent appréciée.

En somme, ce bilan des connaissances démontre la pertinence d'étendre l'évaluation sociale des technologies à la population vieillissante. 
Étienne LaBillois, sociologue diplômé de l'Université Laval.

Nicole Boucher, travailleuse sociale et professeure à l'École de service social de l'Université Laval.

\section{L'univers des personnes âgées et les technologies nouvelles Recension des écrits}

"La mutation vers une société postindustrielle informatisée et vieillissante est aussi celle de la flexibilité et de l'individualisme généralisés, des âges mobiles et des temps incertains. »

$\mathrm{Qu}$ 'on le veuille ou non, l'innovation technologique fait maintenant partie intégrante du quotidien de la société québécoise vieillissante. La course mondiale au développement technologique amorcée après la Deuxième Guerre mondiale, loin d'être terminée, semble s'accélérer en cette fin de siècle. Après la construction des ordinateurs mastodontes durant les années 40 et 50 , le facteur décisif des progrès remarquables des années 80 et, désormais liés, des technologies de l'information et de la communication, des biotechnologies et des technologies industrielles, est la miniaturisation des composants électroniques, elle-même autorisée par l'utilisation de nouveaux matériaux. L'ensemble de cette évolution était encore inimaginable en 1960, de même qu'il reste impossible de prévoir, d'une façon certaine, les progrès techniques futurs et leurs impacts (Schméder, 1988).

De nombreuses études sont menées, depuis plusieurs années, afin de démythifier et de comprendre le phénomène technolo- 
gique et ses conséquences possibles sur nos quotidiens. Il s'agit là $d^{\prime}$ une entreprise ardue. Toutefois, ces recherches ont permis de démontrer que toutes les couches sociales sont affectées, à des degrés divers, par l'expansion technologique. Les impacts sociaux provoqués par la technologie sont nombreux, profonds et envahissants, d'où l'importance d'intensifier, de poursuivre les recherches, au-delà des études de perceptions et d'évolution des tâches, afin de cerner et de comprendre les changements profonds engagés.

En ce qui nous concerne, nous voulons centrer nos travaux sur la population dite âgée. Malgré I'abondance de documents scientifiques sur l'impact social de l'introduction de technologies nouvelles, il semble que ces études soient concentrées dans le secteur du travail et touchent peu les populations dites âgées. Selon McGuire (1986), peu de recherches se sont attardées à mesurer les attentes, les besoins, les attitudes, les perceptions et les conséquences de la technologie sur les activités quotidiennes de nos aînés. Même les études les plus récentes sur les travailleurs âgés et la retraite du BIT (1984), de Danielle Riverin-Simard (1986) sur les étapes de vie au travail, de Xavier Gaulier (1988) sur l'évolution des cycles de vie professionnelle et familiale et le rapport français sur le vieillissement, l'emploi et le travail (Haut Conseil de la population et de la famille, 1989) ne traitent pas de façon particulière cette dimension. Pourtant, il est certain que plusieurs aspects de la vie des travailleurs âgés, des gens du troisième et du quatrième âge risquent d'être affectés par l'innovation technologique. Lesquels ? Comment ? Que savons-nous sur l'influence qu'a l'évolution technologique sur la catégorie de population dite âgée ?

Dans ce contexte, il nous a semblé pertinent et urgent de connaître et d'évaluer les conséquences de l'informatisation et de l'évolution technologique dans l'univers des personnes dites âgées. Nous amorçons cette démarche par une recension des écrits qui, sans être exhaustive, nous permet de relever les dimensions le plus souvent étudiées et les différents angles sous lesquels la technologie a été traitée en gérontologie. Ce bilan des connaissances existantes alimente notre réflexion et facilite le développement $d^{\prime}$ 'un cadre de référence pour de futurs projets de recherche sur l'évolution technologique et le vieillissement au Québec.

Pourtant, nous pouvons d'ores et déjà affirmer que toutes les sphères, tant économiques que politiques et sociales, sont et seront imprégnées par les innovations technologiques. Aucun secteur n'échappe à l'envahissement technologique, qu'il s'agisse du monde du travail, du monde de la santé et des services sociaux, des loisirs, etc. Les individus sont constamment confrontés à de nou- 
veaux appareils sophistiqués et complexes disponibles sur le marché et ce, sans qu'il soit possible de prévoir la fin de ces développements.

En somme, nos modes de vie en sont profondément transformés. On peut penser, comme Proulx (1984: 8), que ces changements technologiques risquent $d^{\prime}$ affecter :

le tissu même de la socialité : de nouveaux modes de connaissances et d'autoconnaissances deviennent possibles, il y a réorganisation des liens sociaux en nouveaux réseaux d'affinités et de solidarités, et autocréation de nouvelles identités personnelles et sociales.

En fait, le fonctionnement de la société québécoise est déjà profondément transformé par l'évolution technologique, de même que par le vieillissement démographique, l'immigration, de même que par la mondialisation de l'économie, de même que par bien d'autres phénomènes connus et inconnus (Tremblay, 1985; Cordell, 1985; CIEST, 1988). Les changements technologiques et sociaux s'influencent mutuellement, puisqu'ils sont indissociables (Boserup, 1981). Ils évoluent rapidement et parallèlement, à un tel point qu'il devient difficile d'affirmer que la technologie est une cause ou une conséquence. De plus, la technologie est devenue une véritable institution sociale et un outil indispensable pour atteindre les buts que la société se donne (Congress of the United States, 1985; McGuire, 1986; Larochelle, 1989). L'internationalisation de la recherche scientifique et technologique (Brainard et al., 1988), combinée à la mondialisation des économies, ne fait qu'accentuer l'appréhension de changements potentiels d'envergure dans le futur. En somme, de nos jours, l'évolution de la société québécoise dépend de phénomènes technologiques, démographiques, économiques et sociaux de plus en plus complexes, qui sont intimement reliés, ce qui n'en facilite pas l'analyse.

Pour ce faire, nous avons sommairement cerné notre sujet de recherche ainsi :

1. les champs d'application de la technologie moderne dans I'environnement des personnes âgées;

2. les attitudes et les perceptions des aînés envers l'informatique;

3. les conséquences de l'introduction de technologies nouvelles dans les secteurs du travail, de la consommation des services de santé, des loisirs et sur les modes de vie.

Afin de tenir compte de la confusion caractérisant les âges, de l'incertitude sur l'âge auquel commencent la vieillesse, le troisième âge et le quatrième âge et de la flexibilité des cycles de vie (Gaulier, 
1988 : 229), nous n'avons pas voulu fixer d'âge chronologique pour identifier la population âgée. La référence aux mots clefs habituels de personnes âgées, d'aînés, de vieillesse et de vieillissement par les auteurs nous sert, dans un premier temps, de base pour repérer les documents pertinents dans les bases de données informatisées. Les mots clefs de technologie et d'informatique leur ont été combinés pour tenter d'appréhender le phénomène dans toute son ampleur. Malgré des recherches dans plusieurs banques de données en gérontologie, en sociologie, en psychologie, en service social, autant américaines que françaises, nous avons trouvé peu de références sous ces mots clefs (85), essentiellement des études américaines, et nous n'en avons retenu qu'une vingtaine pour leur valeur scientifique et leur pertinence.

Le présent article rapporte donc les résultats de cette recherche documentaire. Nous rendons compte des écrits qui existent sur les technologies nouvelles dans l'univers des aînés, en les regroupant sous les thèmes du travail, de la santé, des loisirs et des modes de vie. Mais commençons par préciser, autant que possible, notre terminologie.

\section{Les nouvelles technologies et les personnes âgées : des concepts flous mais utiles}

Pour tenir compte de la diversité et de l'étendue des sens donnés à la notion de nouvelles technologies par les auteurs, nous nous référons à la classification de Maheu et Beauchemin (Tremblay, 1987 : 86-87). Selon eux, trois sens principaux sont associés aux termes de technologies nouvelles. Tout d'abord, elles sont perçues " comme un ensemble plus ou moins complexe, intégré et dense des machines, d'appareils appliqués à la production de biens et de services ". Le second sens leur prête un caractère plus social : "Sont donc constitutifs de machines des manières de faire, des comportements sociaux spécialisés liés à leur utilisation et à leur fonctionnement. Ces comportements sociaux sont appris et transmis, liés à des prescriptions normatives et des valeurs, de même qu'organisés, contrôlés et planifiés ». Enfin, les nouvelles technologies peuvent être perçues "comme un système complexe et organisé de connaissances spécialisées appliquées à la production de biens et de services, au travail de bureau et au traitement de dossiers écrits, voire à la gestion de la société ".

Par ailleurs, elles peuvent prendre trois formes selon le Congress of the United States, (1985). Elles seront douces (soft technology) quand elles s'appliquent à des recherches ou au développement des connaissances. Elles seront fortes (hard technology) 
quand on les utilise pour développer des produits tels que l'équipement médical et de chirurgie (souvent désignés par haute technologie, high technology). Enfin, elles seront faibles (low technology) quand on désigne des systèmes ou produits à utilisation domestique (incluant tous les "gadgets » comme ouvre-boîte automatique, système d'alarme, équipement adapté pour personnes en perte d'autonomie, etc.).

En sommes, nous nous intéressons, dans un premier temps, à tout système automatisé permettant la production de biens et de services, dont le but est d'améliorer la santé et la qualité de vie (tant au travail, à la maison que dans les temps de loisirs) de personnes âgées.

Qu'entend-on par personnes âgées ? Généralement, par la logique de l'analyse démographique, les âges limites de la jeunesse, de l'âge actif et de la vieillesse sont fixés à 15 ans et 65 ans. Consciente que les taux de fécondité et de mortalité faibles conjugués à l'augmentation de l'espérance de vie engendrent l'accroissement relatif des effectifs de plus de 65 ans dans la composition de la population de l'ensemble des pays occidentaux, l'UNESCO prévoit que les personnes âgées atteindront, à l'échelle mondiale, probablement les 600 millions au début du prochain siècle, contre 200 millions en 1950 (UNESCO, 1982). Si le vieillissement démographique est un phénomène commun à presque tous les pays du Nord, on constate de très fortes variations quant à son ampleur et à son calendrier, ce qui rend difficile l'évaluation de ses incidences sur les dépenses sociales et la politique sociale (OCDE, 1988), de même que sur la consommation de technologie (low technology).

Au Canada, cette population âgée de plus de 65 ans s'est accrue de $35 \%$ de 1971 à 1981, contre $13 \%$ pour la population totale, et a atteint un taux de 9,5\%. On prévoit qu'en 2030 cette proportion sera de $20 \%$ avec la génération des baby boomers rendue à la retraite (Driedger et Chappel, 1987). Comparativement aux États-Unis (11,3\%), aux Pays-Bas (11,5\%), à I'Italie $(13,5 \%)$, à la France $(13,9 \%)$, au Danemark $(14,5 \%)$, à la R.F.A. $(15,6 \%)$ et à la Suède $(16,4 \%)$, le Canada reste un des pays les moins touchés par le vieillissement démographique.

Au Québec, le nombre des personnes âgées s'est multiplié par huit de 1901 à 1985, atteignant 634800 personnes, soit 9,6\% de la population totale (Bureau de la statistique du Québec, 1986). Malgré une population vieillissante, le Québec possède encore un des plus bas taux au Canada après Terre-Neuve $(7,7 \%)$ et I'Alberta $(7,3 \%)$. Cette position québécoise avantageuse, qui s'explique par un taux de fécondité assez élevé pendant une bonne période, risque d'être temporaire, car le taux de natalité est maintenant 
l'un des plus bas au monde. Certains prévoient qu'à long terme les aînés pourraient représenter $25 \%$ de la population totale au Québec.

Cette vision des personnes âgées dans la population repose sur une perception généralement admise que la vie de l'individu en société se découpe en différentes strates d'âge, en cycles de vie professionnelle et familiale comportant une hiérarchisation par groupes d'âge. Chacune est constituée de normes et d'obligations qui lui sont propres. Les rôles sociaux des individus sont identifiés et distribués selon leur groupe d'appartenance (Driedger et Chappel, 1987). Cette hiérarchie des groupes d'âge s'appuie inévitablement, sans si ajuster parfaitement, sur le processus, lent et constant, du vieillissement biologique et psychologique des personnes.

Cette méthode statistique pour parler des personnes âgées est insuffisante à notre avis. II est maintenant reconnu que la vieillesse n'est pas uniquement liée à l'âge chronologique, mais également aux règles sociales définissant cet âge. Or, la société fait souvent en sorte que la vie "rentable » des gens s'achève à 65 ans, alors que plusieurs pourraient fonctionner encore des années. Cette règle, légalisée ou non, est déplorable, car les personnes sont presque obligées de cesser leurs activités professionnelles quand cette limite est atteinte (Gaulier, 1984).

Pour nos travaux, sans trancher dans le débat sur les caractéristiques de ces groupes d'âge, nous étendrons la notion de personnes âgées à l'ensemble de la population dite âgée, incluant les travailleurs âgés, les préretraités et les retraités. En fait, nous pensons comme Xavier Gaulier que "l'âge en soi est d'une importance faible, mais les phénomènes de générations, par contre, sont importants ». (1988: 34)

Quoi qu'il en soit, avec cette croissance importante du nombre des aînés, parallèlement à la progression rapide des nouvelles technologies, il y a lieu de s'interroger sur les conséquences de ces dernières sur les gens dits âgés sur les plans du travail, de la santé, des loisirs et des modes de vie.

\section{Le travailleur âgé et les technologies nouvelles}

Dans le monde du travail, plusieurs personnes craignent que la machine atteigne un stade de développement permettant le remplacement de l'homme. Cette crainte est souvent renforcée par un taux de chômage élevé et l'arrivée croissante de nouvelle machinerie (Sauvy et al., 1980). Cette appréhension est d'autant plus forte 
chez les travailleurs âgés qu'ils sont davantage menacés par le chômage (Coutrot, 1986).

Ces derniers résistent ardemment aux déplacements et aux changements apportés dans la sphère de travail. Pour accepter les modifications, ils ont besoin de sécurité et de garanties. Souvent, les changements souhaités par les employeurs sont complexes et ceux-ci ne désirent pas investir dans la formation des travailleurs âgés, d'autant plus que leur efficacité et leur productivité vont en décroissant, sans oublier que leur vie active a une durée limitée (Frossard, 1980).

Pourtant, lorsque apparaissent des changements dans les méthodes de travail, qu'ils soient organisationnels ou techniques, les travailleurs âgés n'ont pas vraiment de difficulté à s'adapter. C'est plutôt la concurrence avec les plus jeunes qui a tendance à les exclure des domaines productifs. Corollairement à cette concurrence entre les générations, la «faiblesse de la formation, de la qualification et des salaires se conjuguent (sic) pour fragiliser la main-d'œuvre [âgée] et participent aux conditions qui permettent son exclusion du système productif (...) »(Frossard, 1980 : 25). Apparaissent dès lors la retraite ou la préretraite qui sont souvent perçues comme un temps de repos exempt de contraintes professionnelles, mais en même temps comme une "inutilité sociale, [un] désœuvrement, [une] marginalisation, [une] 'mort sociale' " (Frossard, $1980: 86$ ).

Si la retraite a un statut ancien et, à ce titre, elle est connue, reconnue et parfois valorisée par la société, par contre la préretraite est récente, dépendante de la conjoncture, changeante et même non prévisible quant à l'âge du retrait et aux conditions de départ. De plus, elle « comporte un certain nombre de risques liés à un changement brutal et imprévu, qui remet en question les équilibres les plus profonds de la personnalité et qui doit faire face à un phénomène nouveau dans l'histoire : l'extension considérable de la durée de la vieillesse »(Gaulier, 1984 : 61). Parce que retraite et préretraite sont deux moyens privilégiés d'exclure les travailleurs âgés du marché du travail, elles affectent l'estime et la confiance en soi des personnes, et entraînent une modification profonde de leurs activités et les contacts interpersonnels. Selon Gaulier, le passage à la retraite provoque une crise d'identité qui "réactive tout le passé et fissure l'avenir » (1984 : 15). Cette nouvelle expérience de la "vieillesse » est l'occasion d'une crise existentielle " résultant d'un conflit intime expérimenté par l'individu entre son aspiration naturelle à la croissance et le déclin biologique et social consécutif à son avancement en âge » (Laforest, 1989 : 47). 
Mais quelles sont précisément les conséquences des progrès techniques et de la transformation des postes et des méthodes de travail sur les travailleurs âgés ? Peu de recherches se sont attardées à vraiment décrire la situation. Par contre, on apprend que selon la théorie de la modernisation de Cowgill (Driedger et Chappel, 1987), les changements provoqués dans la sphère de travail s'appuient sur le déclin du statut et de l'intégration des personnes âgées. L'évolution technologique et son insertion dans le monde du travail accentuent la compétition entre les générations, lutte où les personnes âgées sortent perdantes. On considère, en général, qu'à partir de la quarantaine commencent à se faire sentir les changements qu'apporte la sénescence et que, en conséquence, les travailleurs s'adaptent moins facilement aux changements rapides de la société et aux exigences accrues du patronat. Ainsi, ils se trouvent confinés souvent dans des emplois moins prestigieux et plus sédentaires, sinon condamnés au chômage et à la retraite anticipée.

Même dans le scénario le plus optimiste où l'employeur offre une formation à ses travailleurs âgés afin qu'ils comprennent le fonctionnement d'une nouvelle machine, ceux-ci vont hésiter à s'inscrire pour plusieurs raisons : 1- l'influence de la famille; 2 - la fatigue causée par des soirées d'étude; 3-le doute concernant leur capacité d'assimiler la matière; 4- enfin, le stress de devoir travailler dans un emploi plus complexe.

Selon l'étude du Congrès américain, les aînés sont moins intéressés que les jeunes par l'apprentissage d'une nouvelle machine. En général, ils ont une perception négative des cours de formation et de l'automatisation. Toutefois, il semble que cette perception négative soit moins reliée à l'âge qu'à une question de motivation :

l'appréhension de travailler avec une nouvelle machine, le scep-

ticisme face à ses capacités de recyclage, la résistance face aux technologies nouvelles, les problèmes de retrouver une performance dans une nouvelle façon de travailler, l'impression que l'implication exigée par la tâche limitera leur temps libre, et les difficultés d'accepter les directives d'un jeune instructeur ont tous été identifiés par les répondants comme les attitudes déterminant les limites à la participation de personnes âgées aux formations (Congress of the United States, 1985 : 353, traduction libre).

Le rapport français du Haut Conseil de la population et de la famille sur le vieillissement, le travail et l'emploi tend à confirmer ce point de vue en démontrant l'existence d'un processus d'exclusion des "anciens " parce qu'ils sont considérés comme moins performants (1989: 50). Cette exclusion entraîne prématurément un processus de vieillissement accéléré des travailleurs, qui « varie 
d'une personne à une autre, d'une catégorie socio-professionnelle à une autre, $d^{\prime}$ 'une entreprise à une autre, d'une branche d'activité à une autre...» (1989:57).

Pourtant, ces travailleurs français vieillissants ont des caractéristiques communes dans leur histoire de travail. Ils ont vécu la fin de la société agricole et l'expansion de la société industrielle, tous vivent depuis quelques années le déclin de la société industrielle et notamment des grandes structures de production. Ils sont souvent à l'emploi de la même entreprise où ils ont occupé le même poste ou des postes semblables et n'ont jamais bénéficié de formation professionnelle en cours d'emploi. Tous, notamment dans l'industrie, ont vécu dans un système qui ne tenait pas compte de la dimension humaine et surtout pas du caractère évolutif des aptitudes professionnelles de l'être humain. Faute de flexibilité, ils sont vulnérables parce qu'ils ne sont pas directement adaptables aux nouveaux emplois d'une entreprise créés à la suite de l'introduction d'une technologie plus moderne. Ils sont doublement vulnérables lorsque ces nouvelles technologies entraînent la disparition des postes occupés depuis longtemps. Sans directement étudier les rapports entre l'introduction de technologie et le vieillissement accéléré des travailleurs, ce rapport met nettement en évidence l'existence d'un lien entre l'évolution du travail liée à l'évolution des technologies et la situation des travailleurs âgés.

En somme, on peut penser que les travailleurs âgés risquent d'être les plus touchés par l'introduction d'une innovation technologique dans l'entreprise. Le BIT (1984 : 15) confirme, entre autres, que le progrès technique est une des causes importantes de l'ampleur et de l'aggravation du chômage des travailleurs âgés sur le plan international et de la détérioration de la situation économique et sociale des travailleurs âgés qui représentent un pourcentage important et croissant de la main-d'œuvre. Dans les pays développés, ils sont environ un travailleur sur trois âgés de plus de 45 ans, dont un certain nombre de facteurs de caractère général influencent négativement la situation professionnelle, quels que soient les pays ou les secteurs de travail :

Parmi ces nombreux facteurs qui jouent au détriment des travailleurs âgés, et notamment des plus âgés, on rappellera, en dehors de la concurrence très vive des travailleurs plus jeunes, les difficultés liées au changement des techniques et méthodes, le manque de mobilité et surtout les servitudes psycho-physiologiques dues à l'âge et les idées fausses et préjugés à leur égard. (BIT, $1984: 7$ )

Or, les personnes qui constituent actuellement la masse des travailleurs âgés ont commencé leur vie active il y a plus d'un quart 
de siècle. Leur instruction, leur formation initiale, leurs connaissances de base, générales ou spécialisées, théoriques ou pratiques, appartiennent donc à une autre génération et leur bagage professionnel peut souvent être considéré comme désuet. D'où I'importance d'une formation adaptée au moment de modifications de leur poste de travail liées à l'utilisation d'un appareil nouveau (BIT, 1984 : 23-31).

Au Québec, ces facteurs défavorables ont récemment été traduits par le concept d'employabilité qui, en centrant l'explication sur les problèmes, manques et faiblesses des personnes, permet d'occulter les processus d'exclusion du travail et de chômage prolongé (Provost, 1989). Les modifications récentes apportées au Régime des rentes du Québec en vue d'adoucir les règles d'admission à la rente d'invalidité démontrent pourtant la mise au point de processus d'exclusion des travailleurs usés (Carette et Lamont, 1989).

Au Canada, I'analyse des tendances passées révèle justement que le changement le plus marquant est le déclin de l'activité sur le marché du travail des personnes âgées, manifeste surtout par la tendance nouvelle à prendre une retraite anticipée (Grenier, 1989). De plus, si l'on tient compte qu'au Canada, malgré l'augmentation du nombre de Canadiens âgés de 55-64 ans de 410000 en 1986, ils occupent une part croissante des emplois à temps partiel, de même que le taux de chômage des travailleurs âgés a augmenté entre 1982 $(6,9 \%)$ et $1986(7,3 \%)$ (Akyeampong, 1987), nous pouvons penser que "le virage technologique » des années 80 peut avoir eu une influence déterminante.

Puisque, au Québec, les secteurs en déclin de l'industrie manufacturière, de la construction et du commerce sont ceux où la main-d'œuvre est la plus âgée en 1986 (environ 43 \% des emplois sont occupés par des personnes de 45-64 ans), les risques d'une détérioration future croissante de la situation des âgés " actifs", combinée à une exclusion accélérée, sont élevés. Justement, comment ces observations générales se traduisent-elles dans le quotidien au Québec ? Seules des recherches empiriques dans des entreprises ou des services, à la suite de l'introduction de nouveaux appareils ou de nouveaux systèmes de production, pourront nous permettre de vérifier et de préciser le type de conséquences sociales concrètes vécues par les personnes âgées.

\section{La technologie pour maintenir la santé et prolonger l'autonomie}

Les ouvrages abordant les nouvelles technologies et la santé chez les aînés traitent le problème sous l'angle des dispositifs 
accessibles sur le marché afin d'assurer une qualité de vie minimale aux personnes âgées en leur permettant de vivre en sécurité et de façon autonome à leur domicile.

D'ailleurs, le sens octroyé à la nouvelle technologie est très large. Alors qu'on en parle, en général, comme de systèmes automatisés de production, donc à caractère industriel, une tout autre définition apparait quand on s'intéresse au domaine de la santé. Ici, il existe toute une panoplie d'objets et de techniques permettant l'amélioration de l'état de santé et qu'on considère comme étant des dérivés technologiques. Ils prennent diverses formes, selon le problème qu'on cherche à résoudre.

Ainsi, pour les personnes âgées atteintes de surdité (profonde ou légère) il existe de nouveaux appareils électroniques susceptibles de leur venir en aide (Ryan et Heaven, 1986; Congress of the United States, 1985). On voit apparaître sur le marché des décodeurs électroniques, des systèmes munis d'une imprimante reliée au téléphone, des appareils téléphoniques dotés d'un écran où l'interlocuteur est visible, ce qui permet aux gens atteints d'une surdité légère, et ne pouvant parler sans contact visuel, de dialoguer sans contraintes majeures avec quelqu'un.

D'autres techniques sophistiquées sont également accessibles aux aînés. Il y a le limaçon électronique qu'on place dans le creux de l'oreille et qui convertit les sons en signaux électriques. L'audiophone est également fort répandu chez les aînés. Depuis quelque temps, des techniques ultramodernes sont apparues, mais il est difficile de savoir s'il s'agit là de projets-pilotes ou de matériel facilement accessible aux personnes âgées. Ces techniques électroniques transmettent le son à l'oreille en éliminant toutes les interférences. Elles prennent la forme de bouche auditive (audio loop system), d'une amplification des ondes MF (FM amplifications devices) et de l'amplification des sons à l'aide de l'infrarouge (Congress of the United States, $1985: 84$ ).

En plus de ces mécanismes, des systèmes sont créés pour permettre une vie "normale " aux personnes âgées (ou non) muettes ou aveugles (Ryan et Heaven, 1986). Ainsi, pour celles n'ayant plus l'usage de la voix, il existe un système informatique portatif muni d'un traitement de texte et d'un écran de visualisation permettant le maintien des contacts avec l'entourage. Les personnes aveugles ont maintenant accès à des ordinateurs et imprimantes pour le braille. On trouve d'autres systèmes qui préviennent ces gens en perte de capacité d'un incendie, d'une tentative de vol ou d'une sonnerie téléphonique. Ces systèmes convertissent les sons en signaux visuels. 
II existe également toute une panoplie d'appareils technologiques visant directement l'amélioration de la santé. L'étude du Congrès américain (1985) présente une bonne description de ces possibilités. Outre les mécanismes conçus pour abolir les barrières causées par la surdité, la cécité ou la perte de la voix, les développements technologiques sont également appliqués à la guérison des malaises pathologiques. Par exemple, la technologie permet de résoudre le problème de l'incontinence urinaire. En fait de moyens ou de "technologies", on a créé les couches pour adultes, les cathéters - un procédé par stimulation électrique qui active les muscles en les contractant - et, enfin, les sphincters artificiels. Ceux-ci sont des muscles artificiels installés près de l'orifice urinaire pour resserrer et fermer l'ouverture par un mécanisme de contraction.

Enfin, il y a des systèmes informatiques à caractère plus " domestique » qui sont appelés à jouer un rôle important dans l'amélioration de l'habileté des personnes âgées à demeurer autonomes et indépendantes. Ils renforcent souvent le sentiment de sécurité et la confiance qu'elles ont en leurs capacités.

Dans cette optique, on a des systèmes qui relient la personne âgée à un centre de santé pour prévenir quelqu'un en cas de malaise ou d'urgence (cette liaison peut être établie avec un cabinet de pratique privée, un ami ou un membre de la famille) (Ryan et Heaven, 1986; Congress of the United States, 1985). C'est donc un système de dialogue interactif qui peut prendre deux formes.

La première consiste en un signal de détresse lancé par la personne âgée à un centre de santé à partir d'un petit appareil portatif (qui prendra la forme d'un pendentif ou d'une montrebracelet) quand un problème de santé surgit. Le professionnel de la santé est prévenu et en localisant le signal, il connaît, par son écran, les coordonnées de la personne, les problèmes associés à son état de santé et les soins à apporter. Grâce à ces renseignements, il peut dépêcher sur les lieux une ambulance ou un médecin faisant du service à domicile.

La seconde forme du dialogue interactif est un contrôle effectué par un professionnel de la santé rattaché à un centre médical. Par le système, il expédie régulièrement à la personne âgée (possédant un écran informatique) plusieurs questions auxquelles elle doit répondre afin que le professionnel puisse obtenir une évaluation de son état. Il est possible d'expédier tout simplement un signal à un aîné qui doit répondre en enfonçant un bouton situé sur son pendentif ou sa montre-bracelet. Advenant l'absence d'un retour, un retard important ou toute autre forme de réponse jugée anormale, le système est programmé pour identifier la personne, 
ses coordonnées, l'heure de l'appel et transmettre à un professionnel les données emmagasinées dans son fichier central. À la suite de ce signal, le professionnel peut déterminer les soins appropriés ou la démarche à suivre. Grâce à cette procédure informatisée, les gens âgés sont plus en mesure d'obtenir l'aide nécessaire dans un court laps de temps (Congress of the United States, 1985).

Enfin, il existe des banques de données accessibles aux gens du troisième et du quatrième âge pour obtenir des renseignements divers, tels que les prescriptions pharmaceutiques, l'importance d'une bonne alimentation ou la description d'un programme d'activités (Ryan et Heaven, 1986).

Les technologies décrites précédemment ont été créées et adaptées dans le but de satisfaire les besoins, les désirs et combler les limites des personnes âgées. Mais ces mécanismes répondent également aux besoins de sécurité et de protection tout en veillant à améliorer ou maintenir une bonne santé et une qualité de vie équilibrée. Le Conseil consultatif national sur le troisième âge encourage la création de produits technologiques conçus pour les aînés (CCNTA, 1988). Les aînés bénéficient de possibilités accrues dans l'utilisation de l'ordinateur et de technologies nouvelles, mais leurs attitudes et leurs perceptions envers ces changements ne sont pas encore connues.

\section{Des loisirs technologiques}

Les études ou expériences menées auprès des personnes âgées pour évaluer le degré d'utilisation des nouvelles technologies dans leurs loisirs tiennent compte de deux éléments : les jeux vidéo et l'apprentissage du fonctionnement de l'ordinateur (programmation, banque de données, traitement de texte, chiffrier électronique).

Rien n'est facile à apprendre quand on est âgé, et l'informatique encore moins. Or, le temps joue en faveur des aînés, qui sont souvent enthousiastes face à l'apprentissage de l'ordinateur. Pour eux, cela représente une occasion d'être au fait des derniers développements sur le plan des connaissances et des habiletés, d'établir ou améliorer des relations d'amitié, d'être en mesure de garder contact avec les générations plus jeunes. Ce nouvel apprentissage leur permet de garder l'esprit en éveil tout en évitant de trop réfléchir sur le quotidien, ce qui, en soi, est nuisible (Chin, 1985; Fisher, 1986).

Les chercheurs considèrent l'ordinateur comme un passetemps en expansion, faisant de la concurrence aux activités traditionnelles comme le golf ou l'artisanat (Chin, 1985). Toutefois, 
il semble que les femmes soient davantage intéressées que les hommes à y participer. Fisher (1986) explique ce phénomène par l'aisance des femmes dans les activités sociales, et par une meilleure santé mentale. Le manque d'intérêt des hommes pour les activités liées à l'informatique semble dû à la peur de voir leurs performances évaluées et de paraître malhabiles. Toutefois, Fisher (1986) a remarqué que les hommes vont être plus sensibles aux jeux vidéo lorsque ceux-ci ressemblent aux activités pratiquées dans leur jeunesse, par exemple le " poker».

Même si, au départ, les gens âgés participant à une expérience de formation avaient une véritable phobie de l'ordinateur, ils ont vite compris que l'homme est supérieur à la machine et non I'inverse. À un point tel que désormais " the class members get so involved in the discussion he [the teacher] has trouble shooting them out the door when the class concludes. They love to talk about how they are using their computers and they enjoy sharing information" (Chin, $1985:$ 7). L'enthousiasme est tel que la moitié des personnes âgées d'un centre d'activités de la région de Washington possèdent un ordinateur à la maison.

Plusieurs recherches démontrent que les personnes âgées sont disposées à utiliser l'informatique et qu'elles possèdent les aptitudes nécessaires, malgré le scepticisme de beaucoup d'intervenants. Ces derniers craignent au départ que l'ordinateur soit trop complexe pour être facilement manié quand on est à un âge avancé (Congress of the United States, 1985; Ryan et Heaven, 1986; Fisher, 1986).

Pourtant, on découvre que les réactions sont généralement positives envers l'informatique car il s'agit d'un défi à relever, d'un plaisir jusqu'alors inconnu (Schueren, 1986). Les personnes âgées ont beaucoup de patience, de persévérance et un goût très poussé pour les stimulations intellectuelles (Chin, 1985). C'est pourquoi plusieurs d'entre elles vont préférer monter seules des programmes informatiques au lieu de se contenter de ceux déjà sur le marché.

On ne différencie pas les diverses origines sociales des personnes âgées. Ce qui est différent par contre, c'est l'utilisation qu'on désire faire de l'apprentissage de l'ordinateur. En effet, certaines personnes âgées prévoient utiliser leurs connaissances pour ouvrir un petit commerce, alors que les autres suivent les formations offertes dans le seul but de se distraire. De plus, leurs préférences parmi les activités informatiques varient beaucoup selon les personnes. Certains aînés vont préférer la programmation, alors que d'autres vont privilégier les communications interactives entre usagers, ou encore les jeux vidéo et les logiciels faciles à utiliser. 
Les avantages d'une formation informatique sont nombreux (McGuire, 1984; Hollander et Plummer, 1986; Shueren, 1986). Celle-ci permet aux aînés d'obtenir un environnement plus stimulant, une santé mentale plus équilibrée, une meilleure qualité de vie, un développement accru des habiletés intellectuelles, un degré de concentration supérieur et une adresse perceptuelle améliorée (perceptual-motor skills). La formation est aussi l'occasion de créer de nouvelles amitiés tout en favorisant le divertissement par un climat de compétition amicale (friendly competition).

L'apprentissage de la technologie représente, pour plusieurs, " a way to reach the level of love and belongingness (including needs for acceptance, warmth, and approval) and levels of esteem (including needs for adequacy, worth, status and self-respect)» (Hollander et Plummer, 1986 : 67). Après leur projet, ces auteurs ont effectué un sondage auprès des personnes âgées ayant suivi la formation. Ils ont découvert que $48,5 \%$ des gens voulaient apprendre à utiliser d'autres logiciels, 26,3\% s'intéressaient davantage à la programmation et $24,2 \%$ étaient satisfaits des programmes soumis aux fins du projet sans vouloir nécessairement aller plus loin. Chin (1985) arrive à des conclusions similaires. Elle stipule que le choix d'un passe-temps informatisé n'a pas vraiment d'importance. Ce qui compte avant tout, c'est que les personnes âgées aient du plaisir à l'intérieur de ces activités.

Par contre, Hollander et Plummer (1986) et Schueren (1986) affirment que les aînés ont une préférence marquée pour les jeux vidéo, à condition qu'ils fassent appel à leurs connaissances, à un degré de concentration faible, où la vitesse d'exécution est lente et où les images et les graphiques sont clairs. De plus, les personnes âgées préfèrent les jeux-questionnaires à toute autre forme d'amusement électronique, car elles obtiennent immédiatement les corrections de l'ordinateur par rapport aux réponses fournies.

Ce qui est intéressant dans la participation des personnes âgées à de telles activités, c'est qu'en recherchant un nouveau passe-temps, une façon de communiquer avec les petits-enfants ou une occasion de mettre sur pied une entreprise, elles contribuent à détruire le mythe voulant que les gens arrêtent de fonctionner et deviennent moins utiles en vieillissant (Chin, 1985).

\section{Les modes de vie : la sécurité grâce à la technologie}

Les nouvelles technologies applicables à l'environnement domestique ont continuellement fait l'objet d'améliorations afin d'accommoder les gens âgés et d'accroître leur sécurité. Ces der- 
niers souffrent souvent de divers handicaps, tels que perte d'autonomie, baisse des facultés auditives et visuelles, mobilité réduite, etc. D'où l'importance des petites trouvailles " techniques" qui signifient pour plusieurs l'indépendance et une autonomie sauvegardée.

Les personnes âgées de 65 ans et plus ont souvent une attitude négative envers tout ce qui est informatique, mais cela se dissipe après une première utilisation. Une fois la technologie apprivoisée, elles se familiarisent avec ce nouvel équipement (Ryan et Heaven, 1986). Zeithalm et Gilly (1987) affirment que les personnes âgées ne rejettent pas la technologie; celle-ci doit toutefois être plus avantageuse à l'utilisation que les moyens traditionnels.

Pour améliorer le mode de vie des aînés, Ryan et Heaven (1986) estiment que la technologie doit viser à remplacer, plus qu'à soutenir, l'effort normalement nécessaire pour fonctionner sans problème. C'est pourquoi elle doit éliminer, ou à tout le moins réduire, les nombreuses barrières qui entravent la progression des personnes âgées vers l'obtention d'une bonne qualité de vie. Cela est d'autant plus important que la technologie revêt pour elles un avantage marquant vu les moyens de communication devenus plus fréquents et de meilleure qualité.

La technologie doit en plus être une source primordiale et de premier recours pour améliorer la réalisation des activités quotidiennes. D'où l'importance de faire en sorte que les nouvelles technologies correspondent aux besoins réels des personnes âgées si l'on veut qu'elles soient acceptées comme faisant partie de leur univers (Congress of the United States, 1985).

Quelles sont les technologies qui affectent le mode de vie ? Il y tout d'abord les banques informatisées pour des renseignements relatifs aux agences de voyage, réservations, les bulletins de nouvelles, les jeux vidéo, etc. (Chin, 1985). Il y a également les transferts de fonds électroniques que les personnes âgées utilisent en nombre plus important que celles moins âgées (Gilly et Zeithalm, 1985). Il s'agit pour elles d'une façon intéressante de remplacer les moyens traditionnels, car ils sont plus sûrs (safer) et plus commodes (more convenient) que la procédure habituelle. Par contre, les autres technologies, comme le guichet automatique, sont considérées par les aînés comme étant trop impersonnelles et pas suffisamment sûres. On déplore l'absence de contact avec d'autres personnes et on doute de la sécurité des opérations (Zeithalm et Gilly, 1987).

Kerschner et Chelsvig (1981, cités par Zeithalm et Gilly, 1987) prétendent que les personnes âgées n'utilisent pas les calculatrices, les ordinateurs, les magnétoscopes, les jeux vidéo, la câ- 
blovision et les guichets automatiques. Par la lecture de recherches antérieures, on sait que les ordinateurs et les jeux vidéo sont au contraire utilisés et appréciés des aînés. Pour ce qui est de l'utilisation ou non des calculatrices, des magnétoscopes et du « câble ", il faudrait vérifier le tout de façon empirique.

Dans l'entourage immédiat des personnes âgées, l'étude menée par le Congrès américain (1985) affirme que les nouvelles technologies (au sens large, mais se rapprochant davantage du domaine du design industriel que technologique) peuvent contribuer à l'amélioration des modes de vie. L'étude présente deux situations problèmes où les "nouvelles technologies " peuvent jouer un rôle important.

Il y a, en tout premier lieu, les problèmes dus aux chutes. Pour limiter les dégâts, on propose de recourir aux technologies dites faibles (low technologies) et au design industriel. Ainsi, on suggère que les habitations des personnes âgées aient du tapis dans toutes les pièces, et que les meubles en coin et les angles des murs soient arrondis. On suggère aux aînés d'utiliser une canne et de vivre dans un logement où il y a absence de paliers et d'escaliers. Si la maison est à paliers multiples, on suggère l'élévateur électrique muni d'une chaise et installé dans l'aire d'escalier.

Le second problème en importance est l'incendie. Il existe des détecteurs de fumée équipés de sirène ou de jeux de lumière (flashing lights) qui annoncent aux gens affectés par un handicap visuel ou auditif qu'il y a un danger. On propose également les portes qui résistent au feu (fire-resistant metal doors). Enfin, le Congrès américain (1985) considère que les ouvre-pots (jar cover openers) adaptés, les grappins spéciaux pour prendre des pots ou des plats, les poignées plus grosses pour les gens atteints d'arthrite et les salles de bain adaptées (barres d'appui pour la baignoire et la toilette) font partie de la nouvelle technologie dont nous disposons actuellement. Si cette documentation nous permet d'identifier différents appareils considérés comme des technologies nouvelles adaptées aux personnes âgées, elle nous informe peu sur leurs impacts réels (utilisations, attitudes, perceptions, popularité, etc.).

\section{Conclusion : la société technologique vieillissante, une réalité à découvrir}

Les nouvelles technologies, leurs développements fulgurants et leurs conséquences dans la vie des gens ne peuvent laisser personne indifférent. Plusieurs sphères d'activité des personnes âgées sont maintenant affectées par l'innovation technologique. 
Cependant, des recherches sont nécessaires pour bien cerner et comprendre la réalité au Québec.

Nous avons démontré qu'il est pertinent et possible de saisir adéquatement les applications technologiques et leurs conséquences dans l'univers des aînés, dans un ensemble de secteurs tels que le travail, les loisirs, la vie domestique et la santé.

C'est ainsi que le relevé des écrits, principalement américains, révèle qu'à l'instar des autres groupes d'âge, les personnes âgées sont affectées par les innovations technologiques. Les diverses réactions sont souvent liées à de nombreux facteurs positifs et négatifs, mais en général les aînés s'y adaptent assez bien après quelques utilisations.

Devant le vide reconnu au Québec par rapport à cet objet d'étude, nous envisageons de combler cette lacune. Avec les nombreux développements technologiques prévisibles dans les années à venir, il est permis de croire que l'usage des nouvelles technologies va jouer un rôle de premier plan dans l'existence des personnes âgées. Pour planifier et préparer cette période qui avance à grands pas, il faut accroitre la recherche sur cette problématique.

Une telle étude est primordiale, car les progrès technologiques futurs pourront aider les aînés à demeurer plus longtemps en forme et indépendants. Il est sain d'effectuer des études sur l'ensemble des secteurs touchés afin de posséder des assises solides le jour où des outils de référence seront nécessaires.

Il ne faut pas se leurrer en disant que la société informatisée de demain sera sans problèmes, avec des contacts sociaux solides et une existence paisible et sereine. Au contraire, sans intervention immédiate auprès de nos aînés pour définir les attentes, les besoins et les craintes à l'égard de l'informatisation de leur environnement, il se pourrait qu'ils aient beaucoup de difficulté à s'adapter à la nouvelle société informatisée. L'état de marginalisation dans lequel se confinent les personnes âgées ne peut que s'accroître si des interventions ne sont pas effectuées aujourd'hui pour mieux planifier demain. II faut penser qu'un jour ce sera nous...

\section{Références bibliographiques}

AKYEAMPONG, Ernest B. (1987). "Les travailleurs âgés sur le marché du travail canadien », dans La population active, Statistique Canada, vol. 43, $\mathrm{n}^{\circ} 20: 85-120$.

BIT (1984). Travailleurs âgés et retraités. Genève. 
Boserup, Ester (1981). Population and technological change. A study of long-term trends. Chicago : University of Chicago Press.

Brainard, Robert, Charles LeEDMAN et James Lumbers (1988). Perspectives de politique scientifique et technologique. Paris : OCDE.

Bureau de la statistique du Québec (1986). Statistiques démographiques. Les personnes âgées au Québec. Québec : Publications du Québec.

CARETTE, Jean et Suzanne LAMONT (1989). "L'âge usé : une histoire de travail ", Nouvelles pratiques sociales, vol. 2, $\mathrm{n}^{\circ} 2$.

CHIN, Kathy (1985). "The elderly learn to compute ", Aging, no $348: 4-7$.

CIEST, (1988). STS, Science, technologie et société, sous la direction d'A. Caron et d'A. Michaud. Montréal : Université du Québec à Montréal, Département de philosophie.

Congress of the United States (1985). Technology and aging in America. Washington : Office of technology assessment.

Conseil consultatif national sur le troisième âge (1986). La philosophie du CCNTA concernant la technologie dans une société vieillissante. Ottawa : CCNTA.

CORDELL, Arthur J. (1985). Le grand dérangement, à l'aube de la société d'information. Hull : Conseil des sciences du Canada.

COUTROT, L.R. (1986). "Haute technologie et emploi aux États-Unis", Sociologie du travail, $\mathrm{n}^{\circ} 1$ : 94-113.

DrIEDGER, L. et N.L. ChAPPEL (1987). Aging and ethnicity, toward an interface. Toronto et Vancouver : Butterworths.

FISHER, Sarah (1986). "Increasing participation with a computer in an adult day care settings », Activities, adaptation \& aging, vol. 8, $\mathrm{n}^{\circ} 1: 31-36$.

FrosSARD, Michel (1980). L'emploi des travailleurs âgés et la crise. Le cas français. Grenoble : Université des sciences sociales de Grenoble, Faculté des sciences économiques.

LAFOREST, Jacques (1989). Introduction à la gérontologie, croissance et déclin. Montréal : Éd. Hurtubise HMH.

LAROCHELLE, Gilbert (1989). L'imaginaire technologique. Montréal : Éd. Boréal.

GAULIER, Xavier (1988). La deuxième carrière. Âges, emplois, retraites. Paris : Éd. Seuil.

GAULIER, Xavier (1984). Préretraite, santé, vieillissement. Paris : CNRS, Centre d'études sociologiques.

GiLly, M.C. et V.A. ZEITHALM (1985). "The elderly consumer and adoption of technologies », Journal of Consumers Research, vol. 12, n 3 : 353357.

GRENIER, Gilles (1989). Les travailleurs âgés dans une société vieillissante : tendances, théories et politiques. Ottawa : Université d'Ottawa, Département de sciences économiques.

GUÉRIN, Gilles et Michel HÉBERT (1988). La situation des travailleurs âgés sur le marché du travail au Québec. Montréal : École de relations industrielles, Université de Montréal. 
Haut conseil de la population et de la famille (1989). Vieillissement et emploi, Vieillissement et travail, présenté par Paulette Girard, établi par Élizabeth Lion. Paris : La documentation française.

HOllander, E.K. et H.R. Plummer (1986). "An innovative therapy and enrichment program for senior adults utilizing the personal computer", Activities, adaptation \& aging, vol. 8, $\mathrm{n}^{\circ} 1: 59-68$.

MCGUIRE, Francis A. (1984). "Improving the quality of life for residents of long term care facilities through video games ", Activities, adaptation \& aging, vol. 6, $\mathrm{n}^{\circ} 1: 1-7$.

MCGUIRE, Francis A. (1986). "Computer technology and the aged. Implications and applications for activity programs », Activities, adaptation \& aging, vol. $8, \mathrm{n}^{\circ} 1: 1-4$.

OCDE (1988). Le vieillissement démographique. Conséquences pour la politique sociale. Paris.

PROULX, Serge (1984). "L'informatisation : mutation technique, changement de société ? ", Sociologie et sociétés, vol. XVI, $\mathrm{n}^{\circ} 1: 3-12$.

Provost, Monique (1989). " L'employabilité et la gestion de l'exclusion du travail ", Nouvelles pratiques sociales, vol. $2, \mathrm{n}^{\circ} 2$.

RIVERIN-SIMARD, Danielle (1986). Étapes de vie au travail. Montréal : Éd. Saint-Martin.

RYAN, E.B. et R.K.B. HEAVEN (1986). "Promoting vitality among older adults with computers », Activities, adaptation \& aging, vol. 8, $\mathrm{n}^{\circ} 1: 15-30$.

SAUVY, A. (1980). La machine et le chômage. Le progrès technique et l'emploi. Paris : Dunod.

SCHMÉDER, Geneviève (1988). "Prévision technologique : rétrospective critique ", Futuribles.

SCHUEREN, Betty (1986). "Video games : an exploration of their potential as recreational activity programs in nursing homes ", Activities, adaptation \& aging, vol. 8, $\mathrm{n}^{\circ} 1: 49-58$.

TREMBLAY, Marc-Adélard (1985). Nouvelles technologies et société. Actes du colloque du $45^{\mathrm{e}}$ anniversaire de fondation, Québec, Faculté des sciences sociales de l'Université Laval.

UNESCO (1982). Les jeunes, les personnes âgées et le vieillissement. Paris.

ZEITHALM, V.A. et M.C. Gilly (1987). "Characteristics affecting the acceptance of retailing technologies : a comparison of elderly and nonelderly consumers ", Journal of retailing, vol. 63, n 1 : 49-68. 